
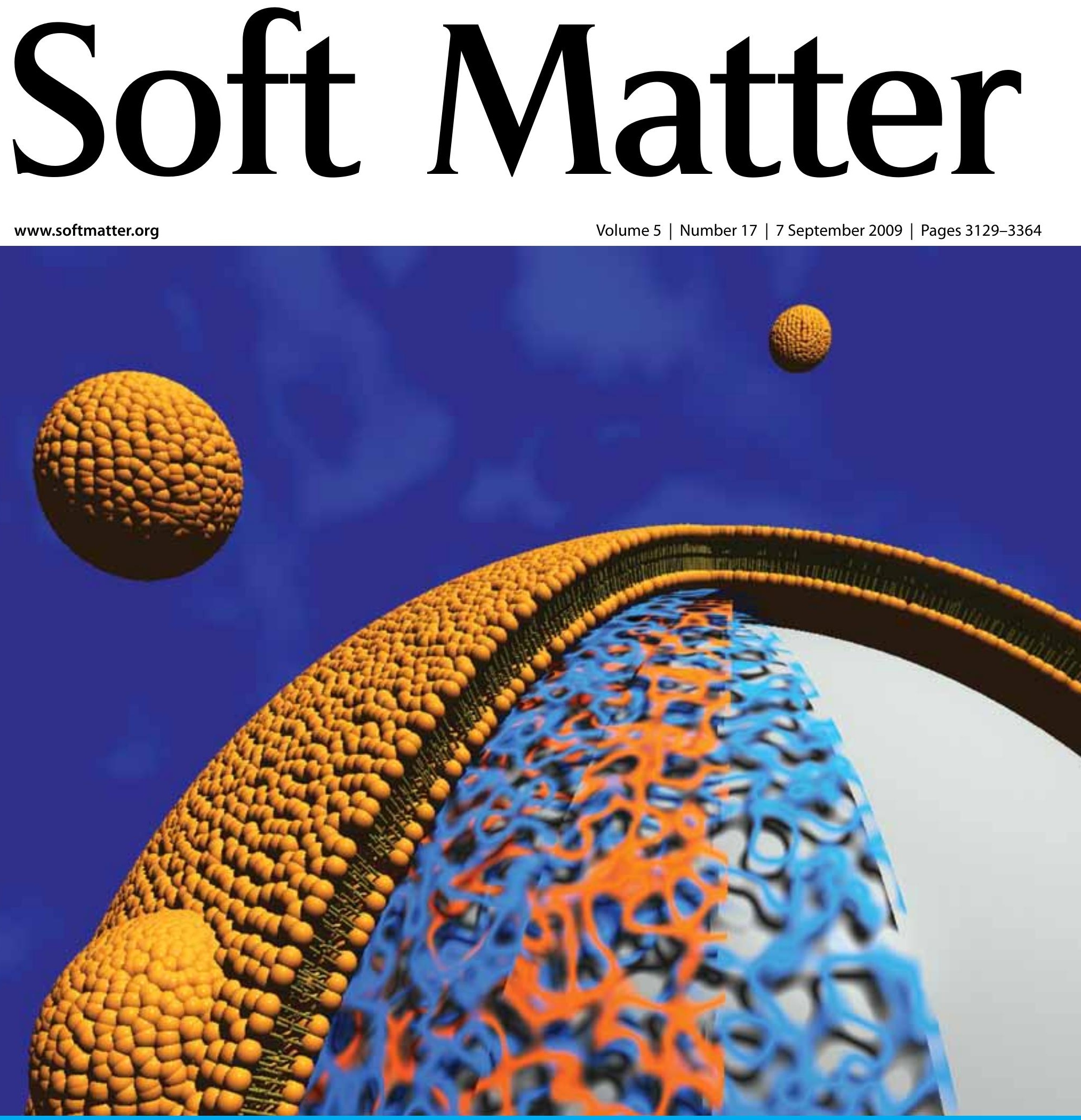

Membrane Biophysics

\section{REVIEW}

Rumiana Dimova et al.

Vesicles in electric fields: Some novel aspects of membrane behavior

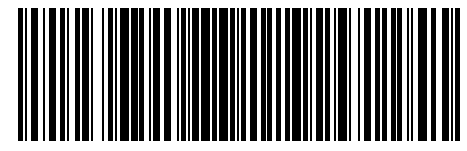

1744-683X(2009)5:17;1-\# 


\title{
Characterization of lipid bilayers adsorbed on spherical LbL-support $\dagger$
}

\author{
Andreas Bunge, ${ }^{* a}$ Martin Fischlechner, ${ }^{b}$ Martin Loew, ${ }^{c}$ Anna Arbuzova, ${ }^{c}$ Andreas Herrmann ${ }^{c}$ \\ and Daniel Huster ${ }^{a}$
}

\author{
Received 3rd February 2009, Accepted 13th May 2009 \\ First published as an Advance Article on the web 25th June 2009 \\ DOI: $10.1039 / \mathrm{b} 902264 \mathrm{c}$
}

Structural and dynamic properties of membranes composed of phosphatidylcholine (PC) and phosphatidylserine (PS) on layer-by-layer (LbL) polyelectrolyte coated particles were investigated using solid-state nuclear magnetic resonance (NMR) and fluorescence methods. These spherically supported membranes showed structural, dynamic, and elastic properties similar to free-standing membranes as proved by ${ }^{31} \mathrm{P}$ and ${ }^{2} \mathrm{H}$ NMR. Small differences between behaviour of PC and PS on LbL support due to interaction with the polyelectrolyte were observed. Fluorescence lifetime imaging microscopy (FLIM) using 7-nitro-2-1,3-benzoxadiazol (NBD) labeled PC and PS showed a stronger impact of the outermost polyelectrolyte (PAH) on the fluorescence lifetimes of NBD-PS compared to NBD-PC. Although small defects in $\mathrm{nm}$ range allowing passage of $\mathrm{Mn}^{2+}$ to both layers of the membrane coat were present, a rather homogeneous coating observed by fluorescence microscopy, complete fluorescence recovery after photobleaching, and NMR results reveal that somewhat continuous lipid bilayers were formed around the LbL particles.

\section{Introduction}

Biocompatible colloids enjoy a tremendously wide application in several scientific, technical, industrial, and medical fields, for instance in tissue engineering, controlled drug delivery, material science, diagnostics, sensorics, and many others. In particular with regard to biomedical applications, soft matter in all conceivable designs is highly relevant and the number of new systems and applications is constantly increasing.

Phospholipid bilayers deposited on a solid support represent a widely-used model system with well-defined geometry and composition to serve basic scientific purposes and numerous (bio-)technological applications. ${ }^{1,2}$ For instance, these systems were used to study lipid membranes, membrane-associated proteins, and protein-ligand interactions. ${ }^{3-6}$ Further, small spherical particles of defined diameter coated with lipid membranes ${ }^{7}$ provide additional advantages, e.g. well-established strategies for straightforward manipulation, handling, and characterization with flow cytometry. ${ }^{8}$ Spherical supported membranes are formed not only on silica beads ${ }^{9}$ but also on layer-by-layer (LbL) polymer coated surfaces. ${ }^{10-12}$ Such LbL particles and capsules are generated by adsorption of anionic and cationic polyelectrolytes on spherical support in an alternating arrangement. ${ }^{13} \mathrm{LbL}$ particles can be easily functionalized, are highly stable, offer a large size range (from $100 \mathrm{~nm}$ to $15 \mu \mathrm{m}$ ), are monodisperse,

${ }^{a}$ Institute of Medical Physics and Biophysics, University of Leipzig, Härtelstr. 16-18, D-04107 Leipzig, Germany.E-mail: andreas.bunge@ medizin.uni-leipzig.de; Fax: +49 (0) 341 9715709; Tel: +49 (0) 341 9715726

${ }^{b}$ Department of Chemistry, University of Cambridge, Lensfield Road, Cambridge, $C B 21 E W, U K$

'Institute of Biology, Humboldt University Berlin, Invalidenstr. 42, D10115 Berlin, Germany

$\dagger$ This paper is part of a Soft Matter themed issue on Membrane Biophysics. Guest editor: Thomas Heimburg. possess a tuneable permeability, and are biodegradable. LbL capsules (particles) carrying reactants or drugs to be released on demand would offer rather novel and attractive applications.

In particular, LbL particles are believed to be relevant for several functions in biomedical research, diagnostics, and therapy and particularly carry high potential for modern approaches in drug delivery. ${ }^{10,11,14}$ In this regard, it has already been shown that LbL particles are sufficiently taken up by living cells, which subsequently express proteins that are encoded on the plasmid encapsulated into the LbL particles. ${ }^{12}$ Typically, the particle uptake occurs in a non-receptor-mediated endocytotic pathway. ${ }^{15,16}$ However, in such applications, the outer polyelectrolyte layer of the LbL particles may give rise to unspecific interactions with numerous molecules found in the body fluids. Therefore, coating of LbL particles with lipid bilayers will allow enhanced stability of the particles, better biological compatibility, more stable encapsulation of drugs or reactants, and controlled release of encapsulated material by reducing the permeability of the LbL shell for polar substances and allowing delivery of drugs into cytosol via fusion of lipid coat with membrane of endosomes. It also allows incorporation of markers, e.g. antibodies, for the specific targeting of the particles $^{17,18}$ and presentation of viralen antigens for easy diagnostics of viral infections using FACS. ${ }^{19}$

Coating of LbL particles with lipid membranes is carried out by mixing the particles with small unilamellar vesicles (SUVs). Typically, the vesicles attach to the polyelectrolyte surfaces and spread spontaneously forming a more or less homogenous bilayer coat. However, large-area coating with intact bilayer membranes represents a delicate task. A recent study on lipid coated LbL particles indicated that the formation of lipid bilayers depends strongly on the surface properties of the support, the biophysical properties of the polyelectrolytes, the ionic strength of the buffer, the roughness of the surface, the hydration state, the operating buffers, and the lipid composition and size of 
the vesicles used for the deposition of the membranes onto the particles. ${ }^{\mathbf{1 0}, 12}$ It has been shown that the best bilayer coating was achieved by using LbL particles composed of poly(allylamine hydrochloride) (PAH) and poly(styrenesulfonate) (PSS) with $\mathrm{PAH}$ as the top layer and $50 \mathrm{~nm}$ diameter vesicles consisting of 1: 1 POPC/POPS lipid mixture. ${ }^{10}$ The headgroups of both phosphatidylcholine (zwitterionic) and phosphatidylserine (negatively charged) may interact with the positively charged polymer PAH, which constitutes the outermost polyelectrolyte layer, where long-range electrostatic attraction of POPS to PAH is essential for supported bilayer formation. The membranes deposited onto the LbL particles, however, do not feature all properties of unperturbed bilayers. For instance, the fluorescence recovery after photobleaching (FRAP) using fluorescently labeled lipids though complete is slowed down: the apparent diffusion coefficient is about 10 times lower compared to diffusion of lipids within freestanding membranes and membranes attached to silica particles. ${ }^{10}$ This apparent slower diffusion might be due to defects in the bilayer, stronger interaction of lipids with the support, and/or larger than calculated surface area coated with lipid. Therefore, studies are required which provide the basis to optimize the properties of the deposited membranes and to prove that the lipid coat on the LbL-particles has the same properties as freestanding membranes.

Here, we characterize the lipid coat (1 : 1 POPC/POPS) on LbL particles with $7 \mathrm{PSS} / \mathrm{PAH}$ layers using solid-state ${ }^{2} \mathrm{H}$ and ${ }^{31} \mathrm{P}$ NMR spectroscopy and fluorescence lifetime measurements. ${ }^{2} \mathrm{H}$ and ${ }^{31} \mathrm{P}$ NMR spectroscopy are very sensitive tools to study the lateral organization, packing, and phase behavior of lipid bilayers. $^{20-22}$ Combined with fluorescence techniques, ${ }^{23}$ a more complete view on the structure and dynamics of the lipid membranes deposited on $\mathrm{LbL}$ particles is generated.

\section{Experimental}

\section{Materials}

Silica particles with a diameter of 3 and $8 \mu \mathrm{m}$ were purchased from microparticles GmbH (Berlin, Germany). Poly(allylamine hydrochloride) (PAH, $700000 \mathrm{~g} / \mathrm{mol}$ ), poly(styrenesulfonate) (PSS, $700000 \mathrm{~g} / \mathrm{mol}$ ) were obtained from Aldrich. The lipids 1-palmitoyl-2-oleoyl-sn-glycero-3-phosphocholine (POPC), 1-palmitoyl- $d_{31}$-2-oleoyl-sn-glycero-3-phosphocholine (POPC- $d_{31}$ ), 1-palmitoyl-2-oleoyl-sn-glycero-3-[phosphor-L-serine] (POPS), and 1-palmitoyl- $d_{31}$-2-oleoyl-sn-glycero-3-[phosphor-L-serine] (POPS- $\left.d_{31}\right)$, 1-palmitoyl-2-[6-[(7-nitro-2-1,3-benzoxadiazol-4-yl)amino]dodecanoyl]-sn-glycero-3-phosphocholine (C12-NBD-PC), 1-palmitoyl-2-[6-[(7-nitro-2-1,3-benzoxadiazol-4-yl)amino]dodecanoyl]-sn-glycero-3-phosphoserine (C12-NBD-PS), 1-palmitoyl-2-[6-[(7-nitro-2-1,3-benzoxadiazol-4-yl)amino]hexanoyl]-snglycero-3-phosphocholine (C6-NBD-PC), 1-palmitoyl-2-[6-[(7nitro-2-1,3-benzoxadiazol-4-yl)amino]hexanoyl]-sn-glycero-3-phospho-L-serine (C6-NBD-PS) were purchased from Avanti Polar Lipids (Alabaster, AL). All lipids were used without further purification.

\section{Methods}

Polyelectrolyte multilayer coating. Silica particles were coated with 7 polyelectrolyte layers starting with PAH using $1 \mathrm{mg} / \mathrm{ml}$ solutions of PAH or PSS in $500 \mathrm{mM} \mathrm{NaCl}$. Each polyelectrolyte was added in excess solution and allowed to adsorb for $10 \mathrm{~min}$ at room temperature. Non-adsorbed polyelectrolytes were removed by 3 centrifugation/redispersion cycles in $100 \mathrm{mM} \mathrm{NaCl}$. Alternating layers of positively and negatively charged polyelectrolytes were deposited. Prior to the addition of lipid SUVs (preparation see below), samples were washed by 3 additional washing steps using $100 \mathrm{mM} \mathrm{NaCl}$.

Preparation of supported lipid membranes. Mixtures of phospholipids were prepared in a chloroform-methanol mixture (1/1 $\mathrm{v} / \mathrm{v})$ (NMR) or chloroform (fluorescence studies). The solvent was removed by rotary evaporation and the resulting lipid film was resolubilized by addition of $100 \mathrm{mM} \mathrm{NaCl}$ and manual swirling of the glass flask resulting in the formation of multilamellar vesicles. The final lipid concentration was $20 \mathrm{mM}$. Subsequently, small unilamellar vesicles (SUVs) were formed by subjecting this suspension to ten freeze-thaw-cycles and extru$\operatorname{sion}^{24}$ through two stacked polycarbonate filters (Nucleopore $\mathrm{GmbH}$, Tübingen, Germany) of $50 \mathrm{~nm}$ diameter using an extruder (Lipex Biomembranes Inc., Vancouver, Canada).

The deposition of the lipid membranes onto the particles was carried out by incubation of the polyelectrolyte coated LbL particles in the presence of SUVs for $60 \mathrm{~min}$ in $100 \mathrm{mM} \mathrm{NaCl}$ at $37^{\circ} \mathrm{C}$ under shaking. Typically, a 10 fold excess of the lipids was used, compared to the calculated amount that is required to obtain the full coverage of the particles with one lipid bilayer. Excess lipids were removed by 5 centrifugation/redispersion steps using $100 \mathrm{mM} \mathrm{NaCl}$. Finally, the solvent was exchanged for buffer solution $(100 \mathrm{mM} \mathrm{NaCl}, 10 \mathrm{mM}$ Tris, pH 7.4 or $100 \mathrm{mM}$ $\mathrm{NaCl}, 10 \mathrm{mM} \mathrm{NaAc}, \mathrm{pH} 5.6$ for NMR or fluorescence studies, respectively) by 2 washing cycles.

For preparation of MLV in the presence or absence of polyelectrolytes, the mixtures of phospholipids were co-dissolved in chloroform-methanol mixture $(1 / 1 \mathrm{v} / \mathrm{v})$. After rotary evaporation of the sample the resulting homogeneous lipid film was hydrated to $20 \mathrm{mM}$ with aqueous solution $(100 \mathrm{mM} \mathrm{NaCl}$, $10 \mathrm{mM}$ Tris, $\mathrm{pH} 7.4$ or $100 \mathrm{mM} \mathrm{NaCl}, 10 \mathrm{mM} \mathrm{NaAc}, \mathrm{pH} 5.6$ for NMR or fluorescence studies, respectively), equilibrated by 10 freeze-thaw cycles and gentle centrifugation. For samples containing polyelectrolytes, MLV were incubated with $\mathrm{PAH}$ solution (in $100 \mathrm{mM} \mathrm{NaCl}, 100 \mathrm{mg} / \mathrm{ml}$ ) with a molar ratio lipid/ PAH 100/1 (mol/mol) followed by 20 freeze/thawing steps. The samples were ultracentrifuged $(100000 \times g)$ over night and the resulting pellets were transferred into $4 \mathrm{~mm}$ high-resolution MAS rotors fitted with spherical Kel-F inserts for liquid samples to prevent samples from drying.

NMR measurements. Static ${ }^{31} \mathrm{P}$ NMR spectra of spherical supported membranes as well of the MLVs were acquired on a Bruker DRX600 NMR spectrometer (Bruker Biospin, Rheinstetten, Germany) at a resonance frequency of $242.9 \mathrm{MHz}$ using a Hahn-echo pulse sequence. ${ }^{25} \mathrm{~A}{ }^{31} \mathrm{P} 90^{\circ}$ pulse length of $7 \mu \mathrm{s}$, a Hahn-echo delay of $50 \mu \mathrm{s}$, a spectral width of $100 \mathrm{kHz}$, and a recycle delay of $3 \mathrm{~s}$ were used. Continuous-wave proton decoupling was applied during signal acquisition. Phosphoric acid was used for external referencing the ${ }^{31} \mathrm{P}$ NMR chemical shift. Spectra were simulated using a program written in MathCad 2001 (MathSoft, Cambridge, USA) considering an elliptical 
distribution of phospholipids due to macroscopic alignment in the external magnetic field.

${ }^{2} \mathrm{H}$ NMR spectra were recorded on a widebore Bruker Avance 750 NMR spectrometer (Bruker BioSpin, Rheinstetten, Germany) at a resonance frequency of $115.1 \mathrm{MHz}$ (magnetic field strength of $17.6 \mathrm{~T}$ ) for ${ }^{2} \mathrm{H}$ using a single channel solids probe with a $5 \mathrm{~mm}$ solenoid coil. The ${ }^{2} \mathrm{H}$ NMR spectra were accumulated using the quadrupolar echo sequence ${ }^{26}$ and a relaxation delay of $1 \mathrm{~s}$. The two $4 \mu \mathrm{s} \pi / 2$ pulses were separated by a $60 \mu$ s delay. Typically, $16 \mathrm{k}$ scans with a spectral width of $500 \mathrm{kHz}$ were acquired. For all experiments, the carrier frequency of the spectrometer was placed in the center of the spectrum. The free induction decays were left-shifted after acquisition to initiate Fourier transformation on top of the quadrupolar echo.

${ }^{2} \mathrm{H}$ NMR spectra were dePaked ${ }^{27}$ and smoothed order parameters ${ }^{28}$ for each methylene group in the chain were determined from the observed quadrupolar splittings $\Delta \nu_{Q}{ }^{(i)}$ for the $i^{\text {th }}$ chain segment according to

$$
\left|\Delta \nu_{Q}^{(i)}\right|=3 / 4 \chi_{Q}\left|S_{\mathrm{CD}}{ }^{(i)}\right|
$$

Here $\chi_{Q}=e^{2} q Q / h$ represents the quadrupolar coupling constant ( $167 \mathrm{kHz}$ for the $\mathrm{C}-{ }^{2} \mathrm{H}$ bond), $S_{\mathrm{CD}}^{i}=1 / 2\left\langle 3 \cos ^{2} \theta_{i}-1\right\rangle$ is the segmental order parameter, $\theta$ is the angle between the bilayer director and the external magnetic field $\mathrm{B}_{0}$.

The Pake doublets were assigned starting from the terminal methyl group exhibiting the smallest quadrupolar splitting. The methylene groups were assigned successively according to their increasing quadrupolar splittings. The smoothed order parameter profiles were determined from the observed quadrupolar splittings as described in detail in Huster et al. ${ }^{29}$ Average order parameters were calculated by adding all chain order parameters and dividing them by the number of methylene and methyl groups in the chain. The chain extension $L_{C}^{*}$ can be estimated by projection of the averaged length of the carbon segments on the bilayer normal and summation over successional carbon segments. $^{30}$

For the ${ }^{2} \mathrm{H}$ NMR relaxation studies a phase sensitive inversion recovery quadrupolar echo pulse sequence was used to obtain the spin-lattice relaxation time $T_{1 Z}$. The ${ }^{2} \mathrm{H}$ NMR spectra were accumulated using a relaxation delay of $2 \mathrm{~s}$. Spectral simulations of the ${ }^{2} \mathrm{H}$ NMR spectra were carried out to obtain the individual $T_{1 \mathrm{Z}}$ by using a program written in MathCad 2001 (MathSoft, Cambridge, USA).

Confocal fluorescence microscopy. C6- or C12-NBD-labeled lipids (NBD-PC or NBD-PS) were added to the lipid mixtures in chloroform to $1 \mathrm{~mol} \%$. All images were taken with an inverted IX81 fluorescence microscope equipped with a Fluoview 1000 scanhead (Olympus, Tokyo, Japan) and a $60 \times($ N.A. 1.35) oilimmersion objective at $25^{\circ} \mathrm{C}$. NBD was excited with the $488 \mathrm{~nm}$ laser line of an Ar-ion laser. The emission of NBD was recorded between 500 to $600 \mathrm{~nm}$.

Fluorescence lifetime imaging microscopy (FLIM). FLIM images were acquired using the time-resolved LSM-upgrade Kit (PicoQuant) mounted on the microscope. NBD was excited at $470 \mathrm{~nm}$ using a pulsed laser diode. The repetition rate of the laser pulse was $10 \mathrm{MHz}$. The fluorescence was detected by a single photon avalanche photodiode with a $(540 \pm 20) \mathrm{nm}$ filter. FLIM pictures were accumulated for 200-300 seconds to obtain a counting rate of about 10000 in the peak channel. The mean count rate was held at maximal 100 counts per millisecond in order to detect on average one photon per 100 laser pulses. The instrument response function (IRF) was recorded for each experimental setup using pure buffer solution, in order to enable iterative reconvolution. Only the lifetime of fluorescence on the surface of a particle was analyzed. The averaged fluorescence decay curve for each particle or MLV was fitted to two- or threeexponential model functions with iterative convolution including a term for scattered light using the Fluofit software (PiqoQuant). Fractional contribution of each decay $f_{\mathrm{i}}$ was calculated using eqn (2). ${ }^{31}$

$$
f_{i}=\frac{\alpha_{i} \tau_{i}}{\sum_{i} \alpha_{i} \tau_{i}}
$$

Here, $\alpha_{i}$ is the amplitude at $t=0 \mathrm{~ns}$ and $\tau_{i}$ is the fluorescence lifetime of the $i^{\text {th }}$ component of the model function.

\section{Results}

\section{Formation of lipid bilayers on LbL surface}

First, the phase state and morphology of the lipids deposited onto LbL particles by spreading of SUVs was investigated using static ${ }^{31} \mathrm{P}$ NMR experiments. A ${ }^{31} \mathrm{P}$ NMR spectrum of POPC/ POPS $(1 / 1 \mathrm{~mol} / \mathrm{mol})$ membranes on $\mathrm{LbL}$ particles of $3 \mu \mathrm{m}$ diameter is shown in Fig. 1A. The general anisotropic ${ }^{31} \mathrm{P}$ line shape of phospholipids in a lamellar phase is characterized by a major peak at high filed and a low field shoulder. The spectrum represents a superposition of two such anisotropic line shapes indicative of two different axially symmetric chemical shift tensors characteristic for the liquid-crystalline lamellar phase state of the membrane that is composed of the two phospholipid species. ${ }^{20}$ At first sight, one might expect that the broader spectral component corresponds to lipids within the leaflet that is localized close to the polymer support while the narrower component is a result of different dynamics within the outer leaflet. However, the pure lipid binary mixture in MLVs without any polyelectrolyte shows a similar spectrum featuring the two anisotropic contributions (Fig. 1C). Therefore, the two spectral components are caused by the different headgroup structure and a slightly different dynamics of POPC compared to POPS in the mixed membrane. The relative integrals of both lines correspond to the correct mixing ratio of the two phospholipids. Such ${ }^{31} \mathrm{P}$ NMR spectra have also been observed for mixtures of POPC and POPG. ${ }^{32}$

The spectra indicate that a lamellar lipid phase of low curvature is formed on the LbL particle surface after spreading of the SUVs. All attached and non-spread SUV are washed away, since such small vesicles would show a narrow line in the ${ }^{31} \mathrm{P}$ NMR spectra at $\sim 0 \mathrm{ppm}$ caused by the fast lateral diffusion of the lipids within the highly curved vesicle surface (Fig. 1D).

To investigate the influence of the PAH in the outer layer of the LbL particle on the membrane morphology, we prepared lipid bilayers composed of POPS and POPC in the presence of PAH. As seen in Fig. 1B, PAH induces only a small amount of 


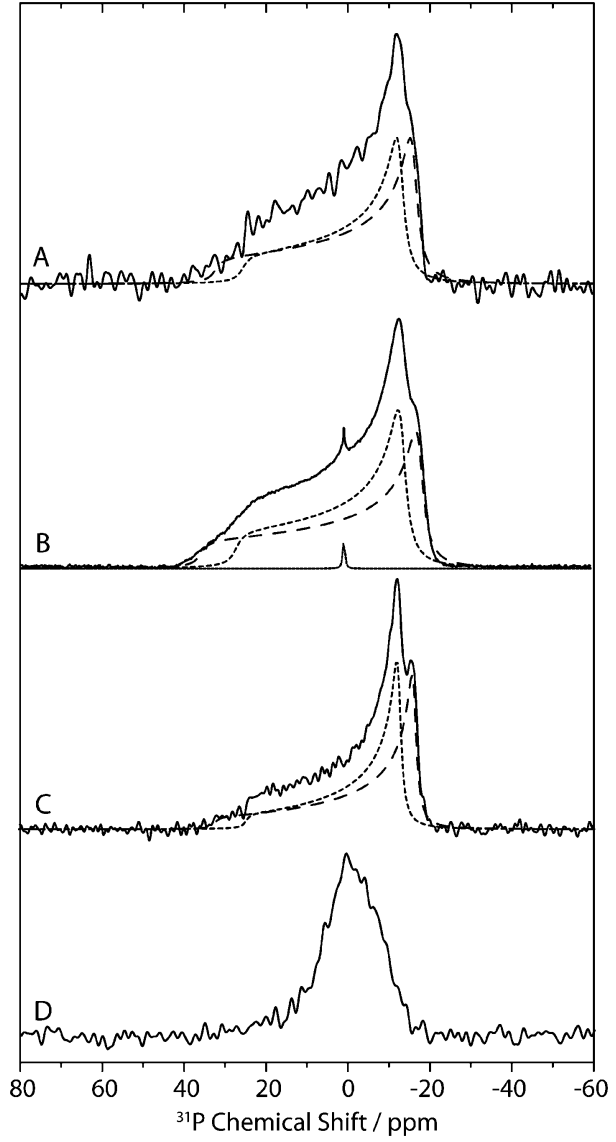

Fig. 1 Proton decoupled 242.9 MHz ${ }^{31} \mathrm{P}$ NMR spectrum of POPC/POPS $(1 / 1 \mathrm{~mol} / \mathrm{mol})$ bilayer on LbL particles (A), multilamellar lipid bilayers in the presence of PAH (B), pure binary multilamellar lipid system (C), and of pellets of SUV after ultracentrifugation (D) recorded at 303K. The dotted and dashed lines indicate the simulated ${ }^{31} \mathrm{P}$ NMR spectra of POPC and POPS, respectively, to fit the experimental line shape.

highly curved non-bilayer lipid phases, since these spectra only contained a very small isotropic signal. Most of the phospholipids remain in the lamellar fluid liquid-crystalline phase.

To compare numbers, the chemical shift anisotropy (CSA, $\Delta \sigma$ ) that is defined as the chemical shift difference between the low- and the high-field edges of the ${ }^{31} \mathrm{P}$ NMR spectrum can be extracted. Alterations in the CSA indicate changes in the orientation or dynamics of the lipid head group. Quantitative values for the ${ }^{31} \mathrm{P}$ NMR chemical shift anisotropy of the two lipids were obtained by using best-fit simulations of the spectrum with two overlapped spectral components and are listed in Table 1.

From these numbers a very similar dynamic behavior of the lipids in the membranes in POPS/POPS MLVs and adsorbed

Table $1{ }^{31} \mathrm{P}$ NMR chemical shift anisotropy values for POPS and POPC in binary mixed membranes acquired at $303 \mathrm{~K}$

\begin{tabular}{lll}
\hline & $\Delta \sigma / \mathrm{ppm}$ (POPS) & $\Delta \sigma / \mathrm{ppm}(\mathrm{POPC})$ \\
\hline Supported lipid membrane & 48.7 & 38.6 \\
MLV in presence of PAH & 53.0 & 41.8 \\
MLV & 48.6 & 37.7 \\
\hline
\end{tabular}

onto the LbL particles can be concluded. In contrast, MLVs prepared in the presence of PAH (POPS/POPS/PAH MLVs), in which most lipid layers might interact with the polymer, show a significant increase in $\Delta \sigma$ for both membrane components. This suggests that the influence of free PAH molecules on the headgroup conformation of the phospholipids is more severe than PAH molecules that are part of the LbL particles. For the three samples there is no indication that the two leaflets of the lipid bilayer experience different membrane environments.

The reduced intensity of the shoulder in ${ }^{31} \mathrm{P}$ NMR spectra for the pure binary lipid mixture is caused by partial magnetic orientation and the induced elliptical shape ${ }^{33}$ of the multilamellar vesicles (Fig. 1C). LbL particles are highly defined in their size and their spherical shape inhibiting macroscopic deformation of the bilayers in the strong external magnetic field. Interestingly, for MLVs prepared in the presence of positively charged polyelectrolytes no orientation effects that would lead to small deviations from the ideal powder distribution of the ${ }^{31} \mathrm{P}$ spectra have been observed. Apparently, the attractive interactions between polyelectrolyte and the lipids are stabilizing the vesicle shape.

\section{Structural properties lipid bilayers on spherical support}

Structural properties of the acyl chains of the individual lipid species in the supported lipid bilayers were investigated using ${ }^{2} \mathrm{H}$ NMR of the lipids with a perdeuterated palmitoyl chain in the membrane. Using either POPS or POPC labeled with ${ }^{2} \mathrm{H}$ (i.e. POPS- $d_{31}$ or POPC- $d_{31}$, respectively) allows determining the properties of each phospholipid in the mixture in an unperturbed fashion. The ${ }^{2} \mathrm{H}$ NMR spectra of POPS- $d_{31}$ or POPC- $d_{31}$ in MLVs formed from the pure binary lipid mixture show well resolved quadrupolar splittings caused by axially symmetric rotations of the lipid molecules characteristic for liquid crystalline phase state (Fig. 2 bottom). The ${ }^{2} \mathrm{H}$ NMR spectra are characterized by a superposition of multiple Pake doublets,
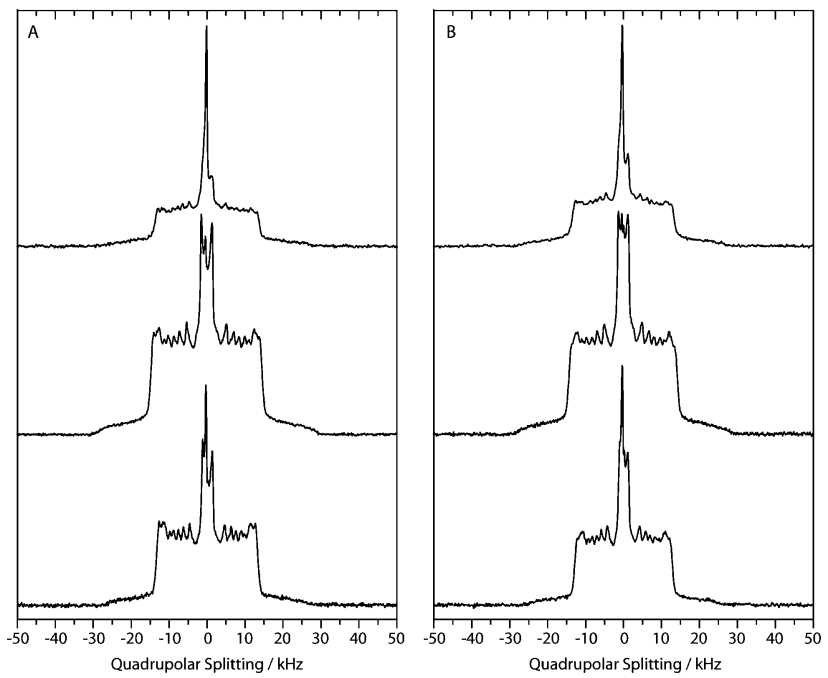

Fig. $2{ }^{2} \mathrm{H}$ NMR spectra of (A) POPS- $d_{31} / \mathrm{POPC}$ and (B) POPS/POPC$d_{31}$ lipid bilayer $(1 / 1 \mathrm{~mol} / \mathrm{mol})$ in POPS/POPC MLVs (bottom), POPS/ POPC/PAH MLVs (middle), and on LbL particles (top) at a temperature of $303 \mathrm{~K}$. 
showing one intense doublet that can be assigned to the terminal methyl groups exhibiting the smallest quadrupolar splitting, 5 well resolved doublets of methylene groups $15-11$ and a plateau region arising from the carbons $2-10$, which cannot be resolved. The isotropic peak in the ${ }^{2} \mathrm{H}$ NMR spectra can be attributed to natural abundance HDO due to the relatively high water content of the samples and is not an indication for highly curved phospholipid structures, since the ${ }^{31} \mathrm{P}$ NMR spectra showed no isotropic signal at $\sim 0 \mathrm{ppm}$. The quadrupolar splittings for POPS are slightly larger than those of POPC in the binary lipid mixture. ${ }^{29}$

Fig. 2 (middle) shows the ${ }^{2} \mathrm{H}$ NMR spectra of a lipid mixture in the presence of excess PAH. For both lipid species, the ${ }^{2} \mathrm{H}$ NMR spectra show increased quadrupolar splittings. It should be noted that for this sample most of the lipid layers are in contact with PAH. There is no indication that the polyelectrolyte induces alterations of the lateral lipid organization in contrast to results reported for highly charged polymers associated with charged membrane surfaces. ${ }^{34}$

The spectra of the lipid bilayers on LbL particles show larger quadrupolar splittings compared to MLVs as well as an additional broadening of the individual doublets. The latter can be attributed to slower lateral diffusion of the phospholipids along the LbL particle with $3 \mu \mathrm{m}$ diameter affecting transverse relaxation times, since $T_{2}{ }^{*}$ decreases with decreasing diameter. ${ }^{7}$ Interestingly, for each lipid species only one set of Pake doublets can be observed in the ${ }^{2} \mathrm{H}$ NMR spectra, indicating that the lipids experience only one fluid environment meaning both lipid layers behave similarly though only inner leaflet is in direct contact with the polymer. ${ }^{35,36}$

To quantify the differences of packing in the hydrocarbon chain region, order parameter profiles were calculated (Fig. 3). As indicated by the shape of the ${ }^{2} \mathrm{H}$ NMR spectra, the order parameters of POPS are only slightly higher than those of POPC in the binary lipid mixture (lower curves). The order parameters of each individual phospholipid in the pure lipid mixture are very close suggesting that the two components are well mixed. PAH interaction with the membrane leads to an increase of the order parameters both for POPS and POPC (upper curves). In the membrane formed on spherical LbL particles the order parameters are slightly increased compared to the pure lipid bilayer (middle curve). The order parameter profiles of each lipid in pure lipid bilayers and on spherical support are very similar for the carbons C10-C16.

Average order parameters and calculated chain extents ${ }^{37}$ are given in Table 2. The averaged order parameters increase for POPS- $d_{31}$ from 0.167 to 0.182 and for POPC- $d_{31}$ from 0.159 to 0.175 when almost all lipids can be in contact with PAH. For the POPS/POPC mixture the difference in average order parameters is 0.008 , while in the POPS/POPC/PAH mixture this difference decreases slightly to 0.007 . From these numbers, chain length differences of $0.2 \AA$ can be calculated. In the LbL particle supported membrane the hydrocarbon chain of POPS- $d_{31}$ shows an averaged order parameter of 0.171 , while POPC- $d_{31}$ features an order parameter of 0.164 that is an intermediate between the values for the pure lipid membrane and the POPS/POPS/PAH MLVs. The negligible differences in the order parameters of POPS and POPC in the pure lipid bilayer, in the presence of the polyelectrolyte, and on the LbL support indicate that the lipids
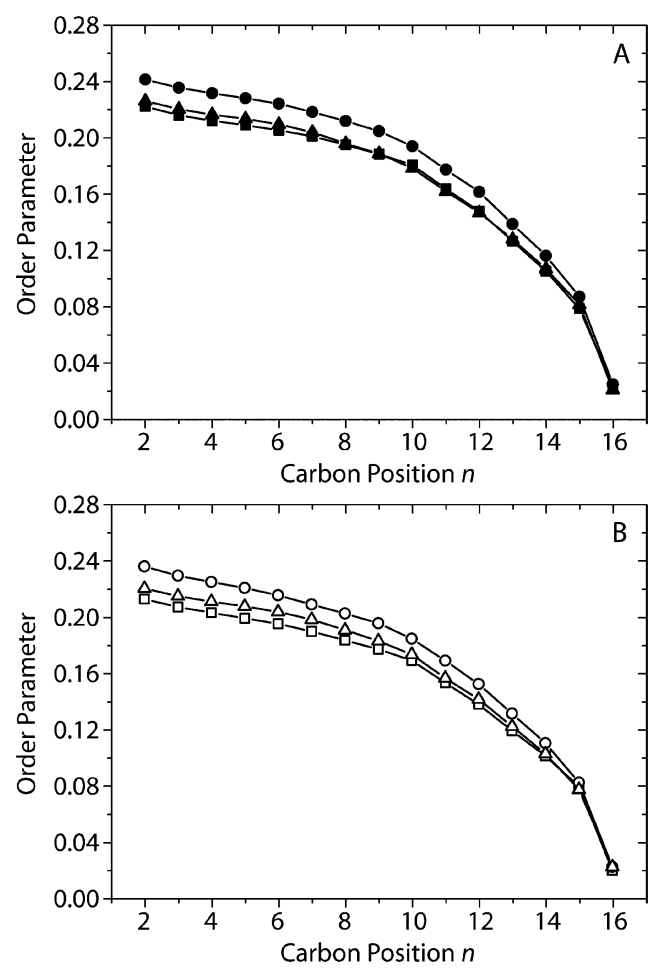

Fig. 3 Smoothed chain order parameter profiles calculated from the ${ }^{2} \mathrm{H}$ NMR spectra of lipid bilayers composed of (A) POPS- $d_{31}$ (filled symbols) and (B) POPC- $d_{31}$ (open symbols) of the samples POPS/POPC MLVs $(\boldsymbol{\square}, \square)$, POPS/POPC/PAH MLVs $(\bullet, \bigcirc)$, and POPS/POPC coat on LbL particles $(\boldsymbol{\Lambda}, \triangle)$.

Table 2 Order parameters and chain lengths determined from ${ }^{2} \mathrm{H}$ NMR data acquired for POPS- $d_{31}$ and POPC- $d_{31}$, respectively at $303 \mathrm{~K}$

\begin{tabular}{|c|c|c|c|c|}
\hline & \multicolumn{2}{|c|}{ POPS- $d_{31}$} & \multicolumn{2}{|c|}{ POPC- $d_{31}$} \\
\hline & $\left\langle\mathrm{S}_{\mathrm{CD}}\right\rangle$ & $\begin{array}{l}\text { chain } \\
\text { extent } / \AA\end{array}$ & $\left\langle\mathrm{S}_{\mathrm{CD}}\right\rangle$ & $\begin{array}{l}\text { chain } \\
\text { extent/A }\end{array}$ \\
\hline POPS/POPC & 0.167 & 11.9 & 0.159 & 11.7 \\
\hline $\begin{array}{l}\text { POPS/POPC in the presence of } \\
\text { PAH }\end{array}$ & 0.182 & 12.3 & 0.175 & 12.1 \\
\hline $\begin{array}{l}\text { POPS/POPC in supported lipid } \\
\text { membrane }\end{array}$ & 0.171 & 12.1 & 0.164 & 11.8 \\
\hline
\end{tabular}

experience similar environments in a homogenously mixed membrane.

To test if the LbL particles are fully covered with lipid membranes, paramagnetic $\mathrm{Mn}^{2+}$ ions were used. For unilamellar vesicles, addition of $1 \mathrm{mM} \mathrm{MnCl}$ to the buffer leads to a quench of the phospholipid ${ }^{31} \mathrm{P}$ NMR signal intensity by $50 \%$ by enhancing $T_{2}$ relaxation mechanisms. ${ }^{24}$ However, for lipid bilayers on LbL particles, signals were diminished almost completely (data not shown). This indicates that phospholipids in both layers are accessible for paramagnetic ions due to small defects in bilayer coverage of LbL particles.

\section{Dynamical properties of phospholipids on spherical support}

Since NMR relaxation is caused by fluctuating electromagnetic fields caused by internal molecular motions of the lipids within 

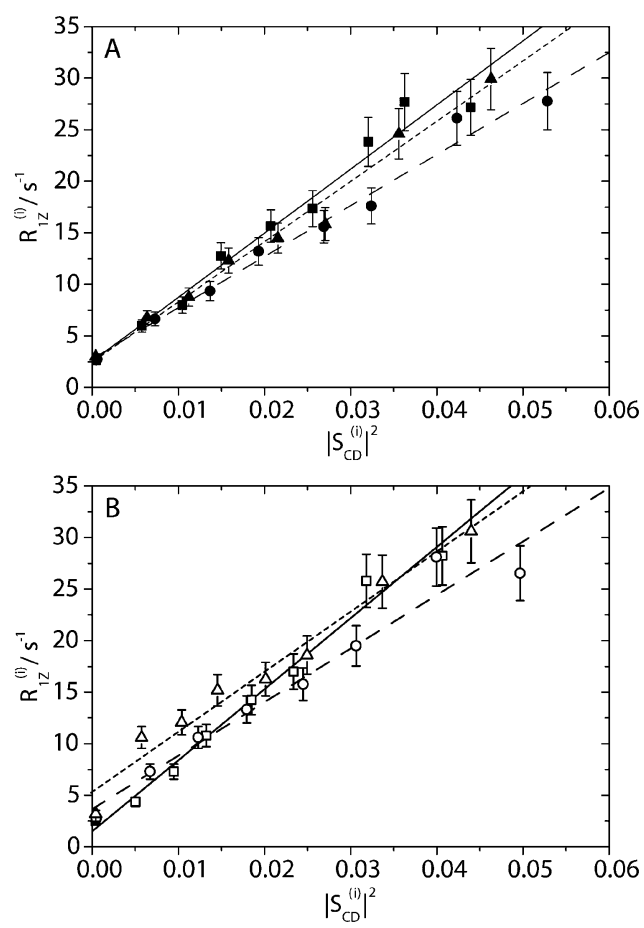

Fig. 4 Square-law plots of lipid bilayers composed of (A) POPS- $d_{31}$ (filled symbols) and (B) POPC- $d_{31}$ (open symbols) of the samples POPS/ POPC MLVs $(\boldsymbol{\square} ; \square$; solid line), POPS/POPC/PAH MLVs $(\boldsymbol{\Delta} ; \triangle$; dashed line), and POPS/POPC on LbL particles ( $\bigcirc$; dotted line). Lines represent linear regressions and are drawn to guide the eye.

the membrane, information about the dynamics of such molecules can be extracted from relaxation measurements. To gain information about the dynamics of the hydrocarbon chains, ${ }^{2} \mathrm{H}$ NMR spin-lattice relaxation measurements on chain-perdeuterated lipids were carried out. For each peak doublet in the ${ }^{2} \mathrm{H}$ NMR powder spectra the Zeeman order longitudinal relaxation time $\left(T_{1 Z}\right)$ was determined in an inversion recovery experiment. The plot of the longitudinal relaxation rates $R_{1 Z}\left(R_{1 Z}=1 / T_{1 Z}\right)$ versus the squared order parameters for saturated lipids typically shows a linear dependence. ${ }^{37,38}$ The slope of such a square-law plot directly correlates with the elastic properties of the membrane. ${ }^{39}$ The square-law plot of POPS- $d_{31} / \mathrm{POPC}$ and POPS/ POPC- $d_{31}$ membranes shows a relatively linear dependence in the pure binary lipid bilayer, in MLVs formed in the presence of PAH and in the coat on LbL particles. In the absence of any polyelectrolyte, POPS- $d_{31} /$ POPC show a slightly reduced slope compared to POPS/POPC- $d_{31}$ bilayers (Fig. 4).

In the presence of $\mathrm{PAH}$ the slope of the square-law plot for POPS of POPC decreases indicating an increased elasticity of the membrane. For bilayers on LbL particles, POPS and POPC show similar square law plots, the slope is slightly decreased compared to the pure lipid bilayer as a consequence of the attachment to the polymer support. This increase in elasticity is less pronounced than in the binary lipid mixture in the presence of PAH.

\section{Interaction of PC and PS with PAH on the LbL surface}

The results from the NMR studies above indicated that the negatively charged POPS when in close proximity to PAH on the

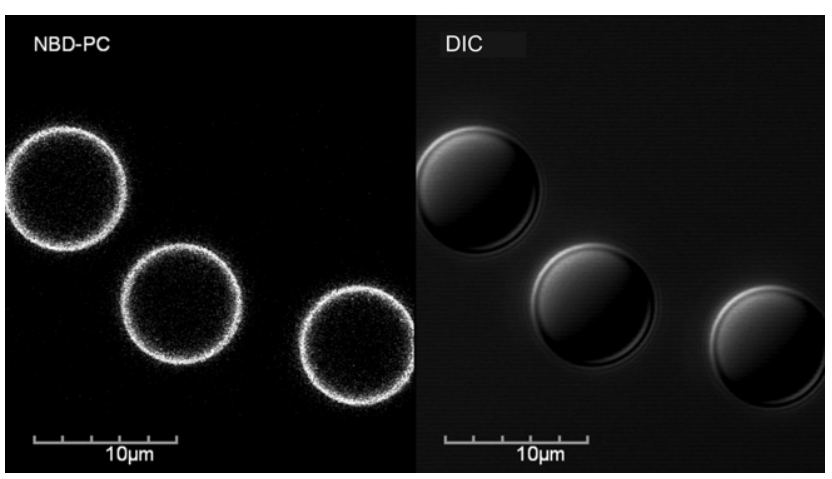

Fig. 5 Fluorescence confocal and differential interference contrast images of POPC/POPS coated LbL particles ( $8 \mu \mathrm{m}$ diameter). Lipid membrane contained $1 \mathrm{~mol} \%$ of C6-NBD-PC.

surface of either LbL-particles or MLVs behaved slightly differently than the zwitterionic lipid POPC.

To study further the influence of PAH on the membrane, fluorescently labeled lipid analogues, NBD-PS and NBD-PC, were used. Fig. 5 shows an image of $8 \mu \mathrm{m}$ LbL particles coated using POPC/POPS SUVs containing $1 \mathrm{~mol} \%$ of C6-NBD-PC. The fluorescence intensity on the surface of the particles was homogeneous and similar for almost all particles indicating homogenous and reproducible coating. Similar results were obtained with C12-NBD-PC. LbL particles coated by using SUVs containing $1 \mathrm{~mol} \% \mathrm{C} 6-$ or C12-NBD-PS were also homogeneously labeled though the intensity was about two times lower. After bleaching at a spot, the fluorescence signal recovered completely for both PC and PS labeled lipid analogues. Similar results were reported previously on $20 \mu \mathrm{m}$ PSS/PAH LbLparticles coated with POPC/POPS/Rh-DOPE membrane. ${ }^{10}$ Complete recovery indicates that lipids in both leaflet of the membrane on the surface of LbL particles were mobile and the membrane had no large defects.

NBD is a polarity sensitive dye: the quantum yield and the fluorescence lifetime are affected by the environment the label is sensing. ${ }^{40}$ Therefore, the influence of PAH and LbL surface on

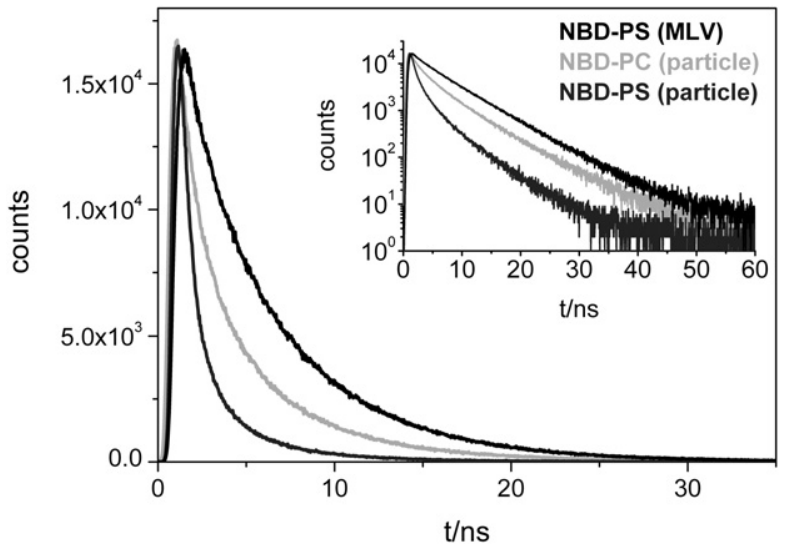

Fig. 6 Typical fluorescence decay curves for C12-NBD-PS in the membrane of POPC/POPS MLVs (black), and C12-NBD-PC (light grey), C12-NBD-PS (dark grey) in the lipid coat on LbL particles $(8 \mu \mathrm{m}$ diameter) measured at room temperature. The insert shows the same data sets plotted on a logarithmic scale. 
Table 3 Fluorescence lifetimes $\tau_{i}$ and their fractional contributions $f_{i}$ of NBD-PC and NBD-PS in MLVs and in the coat on LbL particles measured with FLIM. This is a typical set of data from one day measurements. Lifetimes of ten particles/MLVs were averaged. Errors represent the standard deviations

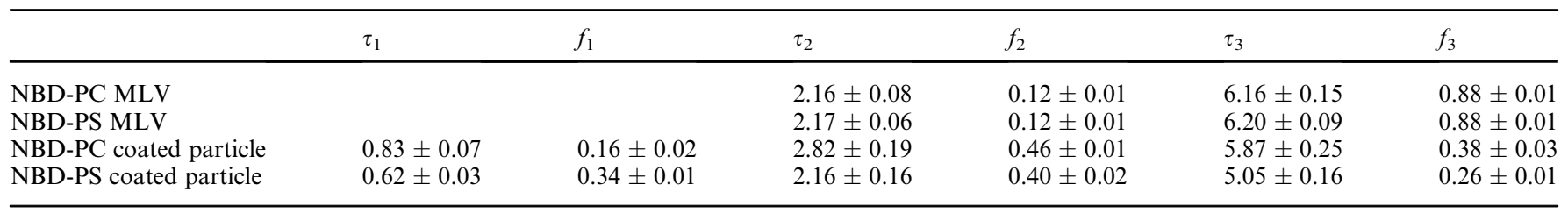

C12-NBD-PC and C12-NBD-PS was compared using fluorescence lifetime image microscopy (FLIM). First we measured the fluorescence decay curves for C12-NBD-PC or C12-NBD-PS in POPC/POPS MLVs, i.e. in the absence of LbL-particle and PAH (Fig. 6, black). Decays could be fitted with two lifetimes of about $2.2 \mathrm{~ns}\left(\tau_{2}\right)$ and $6.2 \mathrm{~ns}\left(\tau_{3}\right)$ with fractional contributions of 0.12 and 0.88 , respectively (Table 3 ), in best agreement with recently reported lifetimes of about $2.5 \mathrm{~ns}$ and $6 \mathrm{~ns}$ for C6-NBD-PC and for C6-NBD-PS in 1/1 DOPC/DOPS giant vesicles, where the longer lifetime is sensitive to physical properties of the membrane such as lipid packing. ${ }^{40}$ We obtained the same results for cuvette experiments using time-resolved fluorescence spectroscopy (data not shown). Therefore, independent of the molecule to which the label is attached (PC or PS), NBD experiences a similar environment in these membranes.

Fig. 6 also shows typical fluorescence decay curves obtained for POPC/POPS coated LbL particles with either C12-NBD-PC (light grey) or C12-NBD-PS (dark grey) in the membrane. Comparing the two decay curves of the coated LbL particles, three observations should be mentioned. First, keeping the duration of the measurements and laser power constant, intensity in the peak channel, though varying from particle to particle, was very similar for both analogues indicating that similar amounts of dye molecules were present in the samples. Second, the area under the decay curves, that corresponds to total intensity, is about half for C12-NBD-PS than for C12-NBD-PC in agreement with microscopic observations. Third, the fluorescence decays are not only different from those observed for MLVs, but also differ between C12-NBD-PS and C12-NBD-PC.

In contrast to POPS/POPC MLVs, three lifetimes were required to fit the decay curves for POPC/POPS/PAH MLVs (not shown) or coated LbL particles indicating a significant change in the environment of NBD due to PAH. We observed two shorter lifetimes, $\tau_{1}$ and $\tau_{2}$ (see Table 3 ), and the longer lifetime $\tau_{3}$, where $\tau_{3}$ was only slightly reduced with respect to $\tau_{3}$ of MLV. We surmise that this lifetime component corresponds to NBD analogues located in the outer leaflet of the bilayer LbLcoat and so not in contact with PAH. Although NBD on the outer leaflet also contributes to $\tau_{2}$, the amplitude of the component $\tau_{2}$ is much higher in comparison to that found for POPS/ POPC MLVs (see Table 3). Thus, we conclude that $\tau_{2}$ as well as $\tau_{1}$ essentially reflects NBD-analogues on the inner leaflet sensing the presence of PAH. The molecular nature of $\tau_{1}$ is not yet clear. One explanation could be that $\tau_{2}$ observed for NBD in the absence of PAH (see Table 3, MLVs) is shifted to $\tau_{1}$ when labeled lipids are close to PAH. Nevertheless, the PAH associated high polar environment causes a significant shortening of lifetimes. In particular, C12-NBD-PS fluorescence was influenced more strongly in comparison to C12-NBD-PC as evident from the shorter lifetimes and higher fractional contributions of the shorter lifetimes (Table 3). Taken together, C12-NBD-PS experiences stronger influence of PAH (polymer support) than $\mathrm{C} 12-$ NBD-PC.

\section{Discussion}

In this study, we combined solid-state NMR and fluorescence life-time measurements to provide a detailed picture of the structure and dynamics of lipid bilayers in the presence of $\mathrm{PAH}$ and on layer-by-layer polymer coated spherical particles.

The ${ }^{31} \mathrm{P}$ and ${ }^{2} \mathrm{H}$ NMR data of this study clearly show that an extended lipid bilayer is formed after attachment of the SUVs to the polyelectrolyte surfaces followed by spontaneous spreading of the vesicles. Fischlechner et al. have shown that the formation of a lipid bilayer on LbL particles is a result of a subtle balance of physical interactions. ${ }^{10}$ Both electrostatic long-range attraction between POPS to PAH and short-range binding via hydrogen bonds between the carboxyl and phosphate group of POPS or the phosphate group of POPC with PAH also contribute to the interaction. The forces are strong enough to cause rupture of the vesicles that interact with the particle surfaces but weak enough to allow successful spreading and deposition of membrane patches, so that a rather intact bilayer can be formed.

Indeed, ${ }^{31} \mathrm{P}$ and ${ }^{2} \mathrm{H}$ NMR spectra of the membrane on the polymer support are very close to those of unperturbed bilayers in vesicles. It has previously been demonstrated that the structural properties of lipid membranes on silica beads without polyelectrolyte coating are similar to those in unperturbed MLVs. ${ }^{9}$ However, the detailed NMR analysis indicates small alterations between the morphological properties of the membranes on the polymer support compared to unperturbed MLVs.

To understand these structural differences we compared NMR parameters of lipid membranes on polymer support with freestanding membranes in the presence of PAH. Polyelectrolytes and phospholipid molecules can form interesting lamellar and non-lamellar phases, which have been characterized in detail. ${ }^{41-43}$ It is known that the interactions between phospholipids and polyelectrolytes affect the structural properties and the dynamics of the lipid membrane. X-ray analysis has demonstrated that membrane-polyelectrolyte aggregates represent closely stacked membranes with the polyelectrolyte sandwiched between the bilayers. Further, NMR studies have shown that (i) polyelectrolyte binding can alter the headgroup orientation of the phospholipid molecules, ${ }^{44,45}$ (ii) increases the packing density of lipid molecules by reducing the area per lipid molecule in the 
membrane, ${ }^{46,47}$ and (iii) may induce domain formation in mixed membranes. ${ }^{48-50}$

Addition of PAH to POPS/POPC membranes affects line shapes of both ${ }^{31} \mathrm{P}$ and ${ }^{2} \mathrm{H}$ NMR spectra of both POPS and POPC with deuterated palmitoyl chains. In the absence of any polyelectrolyte the ${ }^{31} \mathrm{P}$ chemical shift anisotropy for POPC and POPS are $37.7 \mathrm{ppm}$ and $48.6 \mathrm{ppm}$, respectively, in agreement with literature values. ${ }^{34}$ Addition of positively charged polyelectrolytes (PAH) increases the CSA for both lipids as a result of the addition of cationic charges. ${ }^{50}$ The larger relative increase of the CSA for POPS may indicate a stronger interaction with PAH.

Fluorescence lifetime imaging microscopy results show that both C12-NBD-PC and C12-NBD-PS are influenced by PAH. Fluorescence decays of the NBD-analogues became much faster when exposed to PAH. Furthermore, fluorescence lifetimes were even shorter for NBD-PS compared to NBD-PC.

Fluorescence spectra, intensity, and lifetimes of NBD depend strongly on the polarity of the environment. For NBD in water and in chloroform fluorescence lifetimes of 1.4 and 10.1 ns were reported, respectively. Further, the fluorescence intensity is about 4-fold higher in chloroform. ${ }^{51} \mathrm{PAH}$ changes the polarity of the local environment and therefore leads to a reduction of the lifetimes and intensity of NBD lipids situated on the inner leaflet of LbL particles. A higher polarity of the environment due to $\mathrm{PAH}$ can explain the reduction of NBD lifetimes, but not the difference between C12-NBD-PC and C12-NBD-PS. A recent study reported that the head groups of both POPC and POPS interact with $\mathrm{PAH}$, but that PS interaction with $\mathrm{PAH}$ is stronger. ${ }^{10}$ This could lead to the observed differences between C12-NBD-PC and C12-NBD-PS fluorescence lifetimes. It would also explain the lower fluorescence intensity of C12-NBD-PS. The stronger interaction of the analogue with polar neighbors leads to a reduction of the quantum yield.

In agreement, PAH binding to binary lipid mixtures composed of POPS and POPC increases the order in the hydrocarbon chain region in the fluid phase. This ordering occurs over the entire lipid chain, but lipid segments in the upper chain region are more affected indicating that the driving force for the increase in packing density comes from the polar part of the lipid bilayer. ${ }^{52}$

The average area per lipid molecule is determined by an equilibrium of attractive (hydrophobic and van der Waals) and repulsive (electrostatic, steric, and hydration) forces. ${ }^{53}$ The positively charged PAH interacts with the headgroup and possibly the interface region of the membrane, therefore, a perturbation of the lateral force equilibrium in that region is likely. We suggest that the oppositely charged PAH can intercalate between the lipid headgroups and reduce the repulsive forces, which lead to a decrease in the average area per lipid molecule.

As mentioned above, polyelectrolytes may introduce lateral segregation in lipid membranes. Our ${ }^{31} \mathrm{P}$ and ${ }^{2} \mathrm{H}$ NMR spectra do not show any evidence for domain formation induced by $\mathrm{PAH}$, which would cause two resolvable spectral components. This is an indication for a rather homogenous coverage of the bilayer surface with polyelectrolytes. The same conclusion can be drawn from the ${ }^{2} \mathrm{H}$ NMR square-law plots reporting similar dynamic properties of the hydrocarbon chains and bilayer elasticity for POPS and POPC in the presence of PAH.
However, the lipid layer is not fully homogeneous and closed. The results of the $\mathrm{Mn}^{2+}$ assay indicate that ions can easily access all phospholipids from the outer and inner leaflet of the bilayers. This can only be explained by the presence of cavities and distortions of the membrane. A rather homogeneous coating observed by fluorescence microscopy with the resolution of hundreds of nanometre and complete recovery indicate that only small (nm range), if any defects are present in the membrane. Fluorescent microscopy studies reveal that lipid membrane formed on LbL particle support is in close contact with the upper polymer layer, though this does not perturb the membrane continuity.

Taken together, the techniques applied in the current study show that a rather intact lipid bilayer coat can be deposited onto LbL particles with properties that closely resemble those of unperturbed membranes.

\section{Acknowledgements}

This work was supported by a grant from the Federal Ministry of Education and Research (BMBF 0312027 A and D). We thank the DFG (German Research Foundation) and the Experimental Physics Institutes of the University of Leipzig for measuring time the Avance $750 \mathrm{MHz}$ NMR spectrometer.

\section{References}

1 A. A. Brian and H. M. McConnell, Proc. Natl. Acad. Sci. U. S. A., $1984, \mathbf{8 1}, 6159$.

2 M. Tanaka and E. Sackmann, Nature, 2005, 437, 656.

3 L. K. Tamm and H. M. McConnell, Biophys. J., 1985, 47, 105.

4 E. Sackmann, Science, 1996, 271, 43.

5 I. Pfeiffer and F. Höök, J. Am. Chem. Soc., 2004, 126, 10224.

6 K. Glasmästar, C. Larsson, F. Höök and B. Kasemo, J. Colloid Interface Sci., 2002, 246, 40.

7 T. M. Bayerl and M. Bloom, Biophys. J., 1990, 58, 357.

8 A. P. R. Johnston, A. N. Zelikin, L. Lee and F. Caruso, Anal. Chem., 2006, 78, 5913.

9 C. Naumann, T. Brumm and T. M. Bayerl, Biophys. J., 1992, 63, 1314.

10 M. Fischlechner, M. Zaulig, S. Meyer, I. Estrela-Lopis, L. Cuellar, J. Irigoyen, P. Pescador, M. Brumen, P. Messner, S. Moya and E. Donath, Soft Matter, 2008, 4, 2245.

11 C. S. Peyratout and L. Dähne, Angew. Chem., Int. Ed., 2004, 43, 3762.

12 X. Zhang, Q. He, Y. Cui, L. Duan and J. Li, Biochem. Biophys. Res. Commun., 2006, 349, 920.

13 G. Decher, M. Eckle, J. Schmitt and B. Struth, Curr. Opin. Colloid Interface Sci., 1998, 3, 32.

14 F. Caruso, D. Trau, H. Möhwald and R. Renneberg, Langmuir, 2000, 16, 1485.

15 U. Reibetanz, C. Claus, E. Typlt, J. Hofmann and E. Donath, Macromol. Biosci., 2006, 6, 153.

16 M. Semmling, O. Kreft, A. M. Javier, G. B. Sukhorukov, J. Käs and W. J. Parak, Small, 2008, 4, 1763.

17 A. S. Angelatos, B. Radt and F. Caruso, J. Phys. Chem. B, 2005, 109, 3071 .

18 B. Radt, T. A. Smith and F. Caruso, Adv. Mater., 2004, 16, 2184

19 L. Toellner, M. Fischlechner, B. Ferko, R. M. Grabherr and E. Donath, Clin. Chem., 2006, 52, 1575.

20 J. Seelig, Biochim. Biophys. Acta, 1978, 515, 105.

21 J. H. Davis, Biochim. Biophys. Acta, 1983, 737, 117.

22 K. Gawrisch and L. L. Holte, Chem. Phys. Lipids, 1996, 81, 105.

23 K. Suhling, P. M. W. French and D. Phillips, Photochem. Photobiol. Sci. 2005, 4, 13.

24 M. J. Hope, M. B. Bally, G. Webb and P. R. Cullis, Biochim. Biophys. Acta, 1985, 812, 55

25 E. L. Hahn, Phys. Rev., 1950, 80, 580. 
26 J. H. Davis, K. R. Jeffrey, M. Bloom, M. I. Valic and T. P. Higgs, Chem. Phys. Lett., 1976, 42, 390.

27 M. A. McCabe and S. R. Wassall, J. Magn. Reson. B, 1995, 106, 80

28 M. Lafleur, B. Fine, E. Sternin, P. R. Cullis and M. Bloom, Biophys. J., 1989, 56, 1037.

29 D. Huster, K. Arnold and K. Gawrisch, Biochemistry, 1998, 37, 17299.

30 H. I. Petrache, K. Tu and J. F. Nagle, Biophys. J., 1999, 76, 2479.

31 J. R. Lakowicz, in Principles of Fluorescence Spectroscopy, Springer, New York, 3rd edn, 2006, pp. 141-143.

32 D. Huster, X. Yao, K. S. Jakes and M. Hong, Biochim. Biophys. Acta, 2002, 1561, 159.

33 F. Picard, M. J. Paquet, J. Levesque, A. Belanger and M. Auger, Biophys. J., 1999, 77, 888.

34 C. M. Franzin and P. M. Macdonald, Biophys. J., 2001, 81, 3346.

35 A. Bunge, P. Müller, M. Stöckl, A. Herrmann and D. Huster, Biophys. J., 2008, 94, 2680.

36 S. L. Veatch, O. Soubias, S. L. Keller and K. Gawrisch, Proc. Natl. Acad. Sci. U. S. A., 2007, 104, 17650.

37 A. Vogel, C. P. Katzka, H. Waldmann, K. Arnold, M. F. Brown and D. Huster, J. Am. Chem. Soc., 2005, 127, 12263.

38 T. P. Trouard, T. M. Alam and M. F. Brown, J. Chem. Phys., 1994, $101,5229$.

39 D. Otten, M. F. Brown and K. Beyer, J. Phys. Chem. B, 2000, 104, 12119.
40 M. Stöckl, A. P. Plazzo, T. Korte and A. Herrmann, J. Biol. Chem., 2008, 283, 30828.

41 D. D. Lasic, H. H. Strey, M. C. A. Stuart, R. Podgornik and P. M. Frederik, J. Am. Chem. Soc., 1997, 119, 832.

42 J. O. Rädler, I. Koltover, T. Salditt and C. R. Safinya, Science, 1997, 275,810 .

43 I. Koltover, T. Salditt, J. O. Rädler and C. R. Safinya, Science, 1998, 281, 78.

44 C. M. Franzin, P. M. Macdonald, A. Polozova and F. M. Winnik, Biochim. Biophys. Acta, 1998, 1415, 219.

45 D. Huster and K. Arnold, Biophys. J., 1998, 75, 909.

46 D. Huster, U. Dietrich, T. Gutberlet, K. Gawrisch and K. Arnold, Langmuir, 2000, 16, 9225.

47 D. Huster, K. Arnold and K. Gawrisch, Biophys. J., 2000, 78, 3011.

48 P. Mitrakos and P. M. Macdonald, Biochemistry, 1996, 35, 16714.

49 P. Mitrakos and P. M. Macdonald, Biochemistry, 1997, 36, 13646.

50 K. J. Crowell and P. M. Macdonald, J. Phys. Chem., 1998, 102, 9091.

51 K. S. Crowley, G. D. Reinhart and A. E. Johnson, Cell, 1993, 73, 1101.

52 G. Laroche, E. J. Dufourc, M. Pezolet and J. Dufourcq, Biochemistry, 1990, 29, 6460.

53 J. Israelachvili, S. Marcelja and R. G. Horn, Q. Rev. Biophys., 1980, 13, 121. 